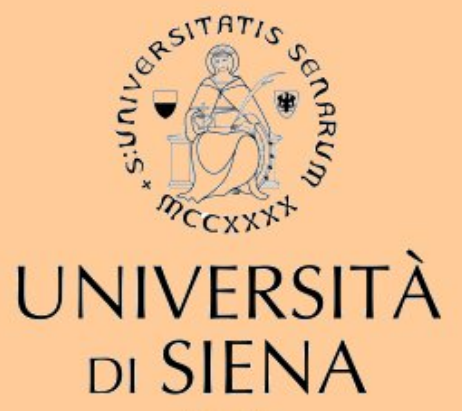

1240

QUADERNI DEL DIPARTIMENTO

DI ECONOMIA POLITICA E STATISTICA

\begin{abstract}
Angelo Antoci Marcello Galeotti

Serena Sordi
\end{abstract}

Environmental pollution as engine of industrialization

n. 725 - Gennaio 2016

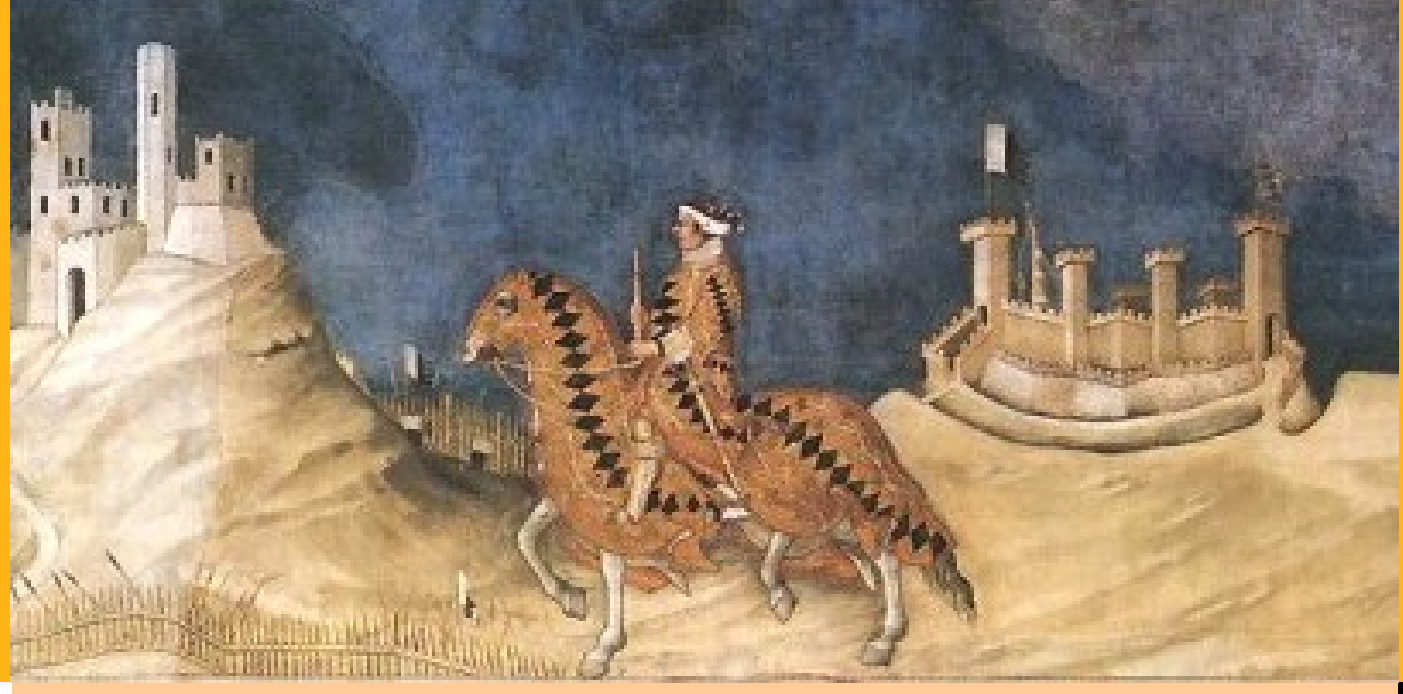




\title{
Environmental pollution as engine of industrialization
}

\author{
Angelo Antoci* \\ Dipartimento di Scienze Economiche e Aziendali, Università di Sassari, via \\ Muroni 25, 07100 Sassari, Italy \\ Marcello Galeotti \\ Dipartimento di Statistica, Informatica, Applicazioni 'G. Parenti', Università di \\ Firenze, viale Morgagni 59, 50134 Firenze, Italy \\ Serena Sordi \\ Dipartimento di Economia Politica e Statistica, Università di Siena, Piazza San \\ Francesco 7, 53100 Siena, Italy
}

\begin{abstract}
This paper analyzes the dynamics of a small open economy characterized by two sectors (a farming sector and an industrial sector), heterogeneous agents (workers and entrepreneurs) and free inter-sectoral labor mobility. Labor productivity in the farming sector is negatively affected by environmental pollution generated by both sectors. Labor productivity in the industrial sector is positively affected by physical capital accumulated by entrepreneurs. We show that, as in the seminal contribution by Matsuyama (1992), low productivity of labor in the farming sector can be an engine of the industrialization process. However, in contrast with Matsuyama's results, our analysis shows that the accumulation of pollution may fuel a self-enforcing process such that the expansion of the industrial sector generates a decrease in workers' revenues.
\end{abstract}

Key words: Two-sector model; structural change; negative externalities; pollution; self-protection behavior.

JEL Classification: D62, E32, C61, B41, E12

\footnotetext{
* Corresponding author

Email addresses: angelo.antoci@virgilio.it (Angelo Antoci), marcello.galeottii@dmd.unifi.it (Marcello Galeotti), serena.sordi@unisi.it (Serena Sordi).
} 


\section{Introduction}

In recent years it has often been argued that industrialization and, more in general, structural changes are both the cause and consequence of economic development and growth (see, e.g., Lewis, 1955; Ranis and Fei, 1961; Lucas, 2004), exactly as happened in Europe in the nineteenth century due to the Industrial Revolution (Bade, 2003). Whatever the cause of structural changes, there is general agreement that they are an integral part of the economic growth process in developing countries and that they produce improvements in the welfare of economic agents. However, an increasing number of contributions in the field deals with the negative impact on welfare of environmental pollution and depletion of free access-natural resources which, in some cases, accompanies structural changes (see, e.g., Lopez, 2007).

The purpose of this paper is to make a contribution to a better understanding of the interactions between environmental pollution, process of industrialization and workers' welfare. To this end, we analyze the dynamics of a small open economy where there are only two sectors (a farming sector, in short, 'F-sector', and an industrial sector, in short, 'I-sector'), free labor mobility and heterogeneous agents (farmers, 'F-agents', and industrial entrepreneurs, 'Iagents'). The latter are characterized as follows. F-agents are endowed only with their own working capacity and use it either in the F-sector, for the production of farming goods, or as employees of I-agents in the I-sector. In turn, only I-agents are able to accumulate physical capital, which is entirely employed in the I-sector to produce, jointly with the labor force provided by F-agents, industrial goods. In our formalization, the state of the economy is described by three variables which are defined as follows. $N \in[0, \bar{N}](\bar{N}-N$, respectively) represents the labor force employed in the F-sector (in the Isector, respectively), $P$, the stock of accumulated pollution and $K$, the aggregated stock of physical capital accumulated by I-agents. Finally, the dynamics of the variables $K, N$ and $P$ are represented by a three-dimensional dynamic system built on a Solow-type capital accumulation mechanism. Labor productivity in the F-sector is negatively affected by $P$, while in the I-sector it is positively affected by $K$.

We show that, as in the seminal contribution by Matsuyama (1992), in such a framework low productivity of labor in the resource-dependent sector can be the engine of the industrialization process. However, differently from Matsuyama, we focus on negative externalities, rather than on positive externalities. In particular, we assume that the production activities of both sectors contribute to an increase in the stock of pollution $P$ and, consequently, generate (ceteris paribus) a reduction in labor productivity in the resource-dependent sector (negative externalities). As a result, if the environmental impact of the I-sector is high enough relative to that of the F-sector, a self-enforcing process 
of industrialization, driven by negative externalities, may be observed. The expansion of the I-sector generates a reduction in labor productivity in the F-sector via an increase in the stock of pollution and therefore leads workers to move from the resource-dependent sector towards the industrial one. The consequent further expansion of the I-sector generates an extra increase in the pollution level from which follows a further reduction in labor productivity in the F-sector, and so on. This expansion of the I-sector, at the expense of the F-sector, may be associated with a decrease in workers' revenues. When this happens - which requires a sufficiently small labor force and a polluting impact of the I-sector higher than that of the F-sector - the transition of labor from the natural resource-dependent sector towards the industrial sector can be classified as a perverse structural change, in the sense of López (2007); namely, a structural change associated with growing problems of environmental degradation, declining or stagnant wages and perpetuation of poverty.

The setup of our model seems to be appropriate in order to describe what happened during the process of industrialization in a number of developing countries. A typical example is given by Haiti, a country which since the 1970s has experimented a development strategy based on export zones for foreignfinanced manufacturing firms (Alscher, 2011; Antoci, Russu, Sordi and Ticci. 2014, p. 221; Collier, 2009). The consequent growing number of these firms and in general of export-oriented apparel industry jointly with very low wages in the agricultural sector has encouraged rural-urban migration, thus increasing the urban population with an extra stress on the environment. As a result, Haiti is now characterized by extreme inequality, low wages, environmental crisis and growing industrialization. Moreover, in other regions which have grown at high rates in recent years, such as small or medium size rural areas of both India and China, environmental degradation is becoming a key issue and citizens are forced to change their behavior to defend themselves against the pollution effects of the industrialization process (see, for example, Economy, 2004; World Bank, 2007; Dhamodharam and Swaminathan, 2010; Boopathi and Rameshkumar, 2011; Deng and Yang, 2013; Holdaway, 2013).

Our model is related to the literature on self-protection strategies adopted by economic agents to defend themselves against the consequences of environmental degradation (see, e.g., Hirsch, 1976; Hueting 1980; Shibata and Winrich, 1983; Bird, 1987; Leipert and Simonis, 1988; Shogren and Crocker, 1991; Yongguan et al., 2001; López, 2003; Escofet and Bravo-Peña, 2007). In the case in which there is no coordination among economic agents, self-protection strategies may give rise to socially undesirable outcomes. The static model proposed by Shogren and Crocker (1991) predicts a level of self-protection higher than the socially optimal one, in the context in which self-protection choices of each economic agent transfer environmental negative externalities to the other agents. In Bartolini and Bonatti (2002, 2003), individuals defend themselves by increasing the consumption of private goods that alleviate the negative 
effects of environmental degradation. In such a case, negative environmental externalities may be an engine of welfare reducing economic growth. In the present paper, workers can defend themselves from environmental degradation by choosing to be employed in the industrial sector (I-sector), and their choices may fuel a welfare reducing industrialization process. The common features of the literature on the undesirable effects of self-protection strategies against environmental degradation are contained in the following description of casual nexuses: $(a)$ environmental degradation stimulates (via self-protection behavior) the expansion of a given sector $X ;(b)$ the consequent expansion of this sector generates further environmental degradation which, in turn, generates a further expansion of the same sector, and so on; $(c)$ the increase in the activity level of sector $X$ may be undesirable; i.e., it may be accompanied by a decrease in welfare; $(d)$ either an exogenous increase of the environmental impact of sector $X$ or an exogenous decrease in the endowment of natural resources have the effect of stimulating the expansion of sector $X$. The feature $(d)$ is shared with the well-known theoretical literature on the curse of natural resources, which has focused on various mechanisms through which the abundance of environmental resources may inhibit growth processes (for a review, see van der Ploeg, 2011). Most current explanations for the curse of natural resources have a crowding-out logic (see Sachs and Warner, 2001): natural resources crowdout the activity of sector $X$; the activity of sector $X$ drives growth; therefore, natural resources harm growth. Sachs and Warner $(1995,1999)$ identify sector $X$ with traded-manufacturing activities; Matsuyama (1992) identifies $X$ with the industrial sector; in Gylfason, Herbertsson and Zoega (1999) and Gylfason (2001), sector $X$ represents education, and so on. In all this literature, the expansion of sector $X$ is always desirable. Indeed, given that it does not generate negative externalities, it always fuels economic growth and leads to an increase in the welfare of economic agents. On the contrary, in the model of the present paper, the development of sector $X$ (the I-sector) may be welfare reducing.

The topic of a structural change driven by low productivity of labor in the resource-dependent sector was also treated in Antoci, Galeotti, Iannucci and Russu (2015) and Antoci, Russu, Sordi and Ticci (2014), although in different contexts. In particular, in both these contributions the labor productivity in the resource-dependent sector was determined by the stock $E$ of a renewable natural resource and was not negatively affected by pollution. ${ }^{1}$ Actually, the assumptions of the present model lead to quite original results in the dynamic

$\overline{1}$ In Antoci et al. (2015), for example, the production technology in the resourcedependent sector was described by the function proposed by Schaefer (1957), widely used in modeling production processes based on the exploitation of natural resources such as fishery and forestry. In the present paper, we will assume a decreasing return technology which in our opinion is more suited to describe production processes in farming. 
analysis, which will be highlighted in the Conclusions.

The structure of the paper is the following. Section 2 introduces the model; Section 3 contains local analysis of the dynamic system; Section 4 investigates global dynamics; Section 5 contains comments about the results of Section 4 and some concluding remarks. A mathematical appendix concludes the paper.

\section{Set up of the model}

In the small open economy with two sectors we model in this paper, the prices of both goods are exogenously determined and, without loss of generality, we assume that they are both equal to unity.

The aggregated production functions of the F- and I-sectors are given, respectively, by:

$$
\begin{array}{rr}
Y_{F}=\frac{\alpha N^{\beta}}{(1+P)^{\gamma}} & 1>\beta>0, \alpha, \gamma>0 \\
Y_{I}=(\bar{N}-N)^{\delta} K^{1-\delta} & 1>\delta>0, \bar{N}>0
\end{array}
$$

The dynamics of the variables $K, N$ and $P$ are assumed to be represented by the three-dimensional dynamic system:

$$
\begin{aligned}
\dot{K} & =s\left[Y_{I}-w(\bar{N}-N)\right]-d K \\
\dot{N} & =\lambda\left(\frac{Y_{F}}{N}-w\right) \\
\dot{P} & =\varepsilon Y_{F}+\eta Y_{I}-\theta P
\end{aligned}
$$

where a dot over a variable indicates the derivative with respect to time.

According to equation (3) - where the difference $Y_{I}-w(\bar{N}-N)$ measures the revenues of I-agents and the parameters $s$ and $d \in(0,1)$, the propensity to save of I-agents and the depreciation rate of $K$, respectively - physical capital is accumulated via a Solow-type mechanism (Solow, 1956). According to equation (4) - where the parameter $\lambda>0$ measures the speed of intersectoral mobility - labor allocation dynamics is determined by the difference between the per capita output in the resource-dependent sector $Y_{F} / N$ and the wage rate $w$ earned in the I-sector, which is assumed to coincide with the marginal productivity of $\bar{N}-N$ :

$$
w=\delta(\bar{N}-N)^{\delta-1} K^{1-\delta}
$$


Finally, the parameters $\varepsilon>0$ and $\eta>0$ in equation (5) represent the effects, on the accumulation of the pollution stock $P$, of the production activities of the F- and I-sectors, respectively, whereas the parameter $\theta$ represents the natural decay rate of $P$.

We assume that the two categories of economic agents take as exogenously given the aggregate outputs $Y_{F}$ and $Y_{I}$ of the two sectors. Thus, in our model, both sectors produce environmentally negative externalities that agents are not able to internalize due to coordination problems. This assumption plays a crucial role in determining the results of the model, much more than the behavioral assumption about the accumulation process of physical capital. In fact, environmental externalities play a crucial role in conditioning economic growth dynamics, especially in developing countries, where environmental resources tend to be less protected and more fragile than in developed countries (López, 2003, 2007).

Given equations (1), (2) and (6), the dynamic system (3)-(5) can be re-written as:

$$
\begin{aligned}
& \dot{K}=s(1-\delta)(\bar{N}-N)^{\delta} K^{1-\delta}-d K \\
& \dot{N}=\lambda\left[\frac{\alpha N^{\beta-1}}{(1+P)^{\gamma}}-\delta(\bar{N}-N)^{\delta-1} K^{1-\delta}\right] \\
& \dot{P}=\varepsilon \frac{\alpha N^{\beta}}{(1+P)^{\gamma}}+\eta(\bar{N}-N)^{\delta} K^{1-\delta}-\theta P
\end{aligned}
$$

In what follows, the dynamics system (7) will be studied in a positively invariant box $\mathcal{B}=\{(K, N, P) \in(0, \bar{K}) \times(0, \bar{N}) \times(0, \bar{P})\}$ after a suitable rescaling. ${ }^{2}$ First of all, we set $K=a K^{\prime}$ such that $s(1-\delta)=d a^{\delta}$, implying, in particular, by renaming $K^{\prime}$ as $K, \bar{K}=\bar{N}$. Then, we pose $K=b K^{\prime}, N=b N^{\prime}$, in such a way that $\alpha b^{\beta-1}=\delta$. Finally we re-scale the time $t$ so as to obtain $\theta=1$. Hence, maintaining the original symbols for the variables, system (7) becomes:

$$
\begin{aligned}
& \dot{K}=l K^{1-\delta}\left[(\bar{N}-N)^{\delta}-K^{\delta}\right] \\
& \dot{N}=m\left[N^{\beta-1}(1+P)^{-\gamma}-(\bar{N}-N)^{\delta-1} K^{1-\delta}\right] \\
& \dot{P}=q N^{\beta}(1+P)^{-\gamma}+r(\bar{N}-N)^{\delta} K^{1-\delta}-P
\end{aligned}
$$

where $l, m, q, r, \gamma, \bar{N}>0,1>\beta, \delta>0$. In fact, in terms of the original

$\overline{2}$ Positively invariant means that the trajectories starting in $\mathcal{B}$ cannot leave it. 
parameters, we have:

$$
l=\frac{d}{\theta}, m=\frac{\lambda \delta}{\theta}, q=\frac{\varepsilon \alpha^{\frac{2}{1-\beta}}}{\delta^{\frac{\beta}{1-\beta}} \theta}, r=\frac{\eta \alpha^{\frac{1}{1-\beta}} d^{\frac{1-\delta}{\delta}}}{[\varepsilon(1-\delta)]^{\frac{1-\delta}{\delta}} \delta^{\frac{1}{1-\beta} \theta}}
$$

while the new $\bar{N}$ is equal to the former one multiplied by $b^{-1}=(\delta / \alpha)^{1 /(1-\beta)}$.

Hence, in the box $\mathcal{B}, \bar{K}=\bar{N}$. In order to determine $\bar{P}$, we proceed as follows. Consider the equation $\dot{P}=f(K, N, P)=0$. Then, as $\frac{\partial f}{\partial P}<0$ for any positive triad $(K, N, P)$, it follows that $f(K, N, P)=0$ defines an implicit function $P(K, N)$ on the open square $(0, \bar{N})^{2}$. But it is easily checked that $P(K, N)$ can be continuously extended to the closed square $[0, \bar{N}]^{2}$. Therefore we define

$$
\bar{P}=\max _{[0, \bar{N}]^{2}} P(K, N)
$$

In this way, it is easily seen that when $(K, N) \in(0, \bar{N})^{2}$, then $f(K, N, \bar{P}) \leq$ 0 and $f(K, N, \bar{P}+\varepsilon)<0$ for any arbitrarily small $\varepsilon>0$. So the box $\mathcal{B}=(0, \bar{N})^{2} \times(0, \bar{P})$ satisfies our requirements.

\section{Local analysis}

Let us consider the function:

$$
\varphi(K)=q(\bar{N}-K)+r K-(\bar{N}-K)^{\frac{\beta-1}{\gamma}}+1
$$

defined for $K \in[0, \bar{N})$. Then, the local analysis results are summed up in the following theorem.

Theorem 1 Consider the above function $\varphi(K)$. Then:

(1) If:

$$
\varphi(0)=q \bar{N}-\bar{N}^{\frac{\beta-1}{\gamma}}+1>0
$$

there exists exactly one stationary state in $\mathcal{B}$, which is a sink.

(2) If $\varphi(0)<0$, there may exist, generically, two or zero stationary states in $\mathcal{B}$. In the former case, named $Q^{*}=\left(K^{*}, N^{*}, P^{*}\right)$ and $\tilde{Q}=(\tilde{K}, \tilde{N}, \tilde{P})$, $0<K^{*}<\tilde{K}<\bar{N}$, the two stationary states, $Q^{*}$ is a saddle endowed with a two-dimensional stable manifold and $\tilde{Q}$ is a sink. In the bifurcation case $Q^{*}=\tilde{Q}$, the stationary state is a saddle-node. 
(3) If $\varphi(0)=0$ and $\varphi^{\prime}(0)>0$, there exists exactly one sink in $\mathcal{B}$; if, instead, $\varphi(0)=0$ and $\varphi^{\prime}(0) \leq 0$, there is no stationary state in $\mathcal{B}$.

PROOF. See Appendix A.1.

Remark 2 From the above theorem it follows that no Hopf bifurcation can occur.

Remark 3 At an interior sink $\tilde{Q}=(\tilde{K}, \tilde{N}, \tilde{P})$ we have $\tilde{N}=\bar{N}-\tilde{K}, \tilde{P}=$ $(\bar{N}-\tilde{K})^{\frac{\beta-1}{\gamma}}-1$, where $\tilde{K}$ is a solution of $\varphi(K)=0$ with $\varphi^{\prime}(\tilde{K})<0$. Therefore, writing $\varphi(\tilde{K}, q, r)=q(\bar{N}-\tilde{K})+r \tilde{K}-(\bar{N}-\tilde{K})^{\frac{\beta-1}{\gamma}}+1=0$, $0<\tilde{K}<\bar{N}$, and assuming $\frac{\partial \varphi}{\partial \tilde{K}}=-q+r-\frac{1-\beta}{\gamma}(\bar{N}-\tilde{K})^{\frac{\beta-1-\gamma}{\gamma}}<0$, we can obtain $\tilde{K}$ as an implicit function, i.e. $\tilde{K}=\tilde{K}(q, r)$, with both $\frac{\partial \tilde{K}}{\partial q}$ and $\frac{\partial \tilde{K}}{\partial r}$ positive. So, at the sink $\tilde{Q}$, when existing, $\tilde{K}$ and $\tilde{P}$ increase, while $\tilde{N}$ decreases with $q$ and $r$. On the other hand, it is easily checked that the existence of two stationary states (the saddle and the sink) implies $q<r$.

Given the expressions for $q$ and $r$ in (9), it follows that their values are (ceteris paribus) positively proportional to $\varepsilon$ (the parameter measuring the environmental impact of the F-sector) and $\eta$ (the parameter measuring the environmental impact of the I-sector), respectively. According to condition (11) in Theorem 1, one (and only one) stationary state exists if (ceteris paribus) either the size $\bar{N}$ of the population of workers or the parameter $q$ are high enough. According to Remark 3, a necessary condition for the existence of two stationary states (the saddle and the sink) is $q<r$; such a condition is satisfied if (ceteris paribus) the parameter $\varepsilon$ is low enough with respect to the parameter $\eta$.

The parameters $q$ and $r$ play a crucial role also in determining the coordinates of the $\operatorname{sink} \tilde{Q}$, when it exists (see Remark 3 ). An increase either in the value of parameter $q$ or in the value of parameter $r$ generates an increase in capital accumulation $\tilde{K}$ and a decrease in the employment level $\tilde{N}$ in the F-sector. The mechanism giving rise to such a result is rather intuitive: an increase in either $q$ or $r$ increases (ceteris paribus) the pollution level $P$ reducing labor productivity in the F-sector; this, in turn, has the effect of increasing labor employment and capital accumulation in the I-sector. In this sense, we can say that, in our model, environmental degradation can be an engine of industrialization, i.e., of a structural change. In the next section we will give a complete classification of the dynamic regimes that can be observed under our three-dimensional dynamic system (8). The global analysis results which are there contained will allow us, among other things, to illustrate the role 
played by the initial value of the pollution level $P$ in determining the future evolution of the economy.

\section{Global analysis}

In this section we will illustrate the whole global dynamics of $(8)$ in $\mathcal{B}$. First of all we prove the following theorem.

Theorem 4 Assume $\varphi(0)<0$. Let $\mathcal{A} \subseteq \mathcal{B}$ be a positively invariant region without stationary states and suppose $\hat{Q}=(0, \bar{N}, \hat{P}) \in \partial A,{ }^{3}$ where $\hat{P}$ is the solution of the equation $q \bar{N}-P(1+P)^{\gamma}=0$. Then all the trajectories starting in $\mathcal{A}$ tend, as $t \rightarrow+\infty$, to $\hat{Q}$. Moreover, if $\mathcal{A} \subset \mathcal{B}$, then the boundary of $\mathcal{A}$ contains the two-dimensional stable manifold of the saddle $Q^{*}$.

PROOF. Given the assumptions of the theorem, if, by contradiction, a trajectory $\Gamma(t)=(K(t), N(t), P(t))$ starting in $\mathcal{A}$ does not converge to $\hat{Q}$, then it keeps oscillating. In particular $K(t)$ will reach a maximum, say, at $t_{1}$. Then $\ddot{K}\left(t_{1}\right) \leq 0$ implies $\dot{N}\left(t_{1}\right) \geq 0$. In fact, since the existence of oscillating trajectories is an open condition, we can assume $\dot{N}\left(t_{1}\right)>0$. Hence $N(t)$ would, in turn, reach a maximum before $K(t)$ reaches a minimum. Suppose that this occurs at $t_{2}>t_{1}$. Then, as $\dot{K}\left(t_{2}\right)<0, \ddot{N}\left(t_{2}\right) \leq 0$ implies $\dot{P}\left(t_{2}\right)>0$. Therefore we can set $t_{2}=0$, so that, in a right neighborhood of $t=0, \dot{K}(t)$, $\dot{N}(t)<0, \dot{P}(t)>0$. Now, consider, as above, the function $P(K, N)$ implicitly defined by $\dot{P}=f(K, N, P)=0$ when $(K, N) \in(0, \bar{N})^{2}$, which can be continuously extended to the closed square $[0, \bar{N}]^{2}$. Then it is easily checked that, for any $K_{0} \in(0, \bar{N})$, the graph of $P\left(K_{0}, N\right), N \in[0, \bar{N}]$, has a parabolic shape, with $P\left(K_{0}, 0\right)=\bar{N}^{\delta} K_{0}^{1-\delta}$ and $P\left(K_{0}, \hat{N}\right)=\hat{P}$. The maximum value $P_{\mu}\left(K_{0}\right)$ is given by the solution of the system $f\left(K_{0}, N, P\right)=\frac{\partial f}{\partial N}\left(K_{0}, N, P\right)=0$ : hence it is easily checked that $P_{\mu}\left(K_{0}\right)$ is increasing with $K_{0}$ and $\lim _{K \rightarrow 0^{+}} P_{\mu}\left(K_{0}\right)=\hat{P}$. On the other hand, set $P_{\nu}\left(K_{0}\right)=\left(\bar{N}-K_{0}\right)^{\frac{\beta-1}{\gamma}}-1,0<K_{0}<\bar{N}$, i.e., $P_{\nu}\left(K_{0}\right)$ is the $P$-coordinate of the intersection $\left\{\dot{K}=\dot{N}=0, K=K_{0}\right\}$. Clearly $\frac{d P_{\nu}}{d K_{0}}>0$. Since we assumed $\varphi(0)<0$, it follows from straightforward computations that $\lim _{K \rightarrow 0^{+}} P_{v}\left(K_{0}\right)=P_{v}(0)=(\bar{N})^{\frac{\beta-1}{\gamma}}-1>\hat{P}$. Hence, let us go back to the trajectory $\Gamma(t) \subset \mathcal{A}$ for $t \geq 0$. Then, if $K_{0}$ is sufficiently small, $P_{\mu}\left(K_{0}\right)<P_{\nu}(0)$. It follows that, since $\dot{K}(t)<0$ in a right neighborhood of $t=0$, a possible maximal value of $P(t)$, say $P\left(t^{*}\right), t^{*}>0$, will

3 A positively invariant region is an open connected set such that all the trajectories starting in it remain there for all $t \geq 0$. By $\partial \mathcal{A}$ we denote the boundary of $\mathcal{A}$. 
satisfy $P\left(t^{*}\right)<P_{\nu}(0)<P_{\nu}\left(K^{*}\right)$. Therefore $K(t)$ keeps decreasing and in fact this implies $\lim _{t \rightarrow+\infty} K(t)=0 .{ }^{4}$ Consequently $\lim _{t \rightarrow+\infty} N(t)=\bar{N}$ and $\lim _{t \rightarrow+\infty} \dot{N}(t)=0$, so that, finally, $\lim _{t \rightarrow+\infty} P(t)=\hat{P}$. Therefore we have proven that, if $Q_{0}=\left(K_{0}, N_{0}, P_{0}\right) \in \mathcal{A}$ and $K_{0}$ is small enough, then the trajectory through $Q_{0}$ converges, as $t \rightarrow+\infty$, to $\hat{Q}$. Now, suppose that the trajectory starting at some $Q_{0} \in \mathcal{A}$, with, as above, $\dot{K}\left(Q_{0}\right)<0, \dot{N}\left(Q_{0}\right)=0$, $\dot{P}\left(Q_{0}\right)>0$, reaches $\dot{K}=0$ before $\dot{N}=0$. Then, by the continuous dependence of the solutions from the initial conditions, there must be some $Q_{0}$ whose trajectory reaches a point $Q^{*} \in\{\dot{K}=0\} \cap\{\dot{N}=0\}$. Moreover, again by a continuity argument, ${ }^{5} \dot{P}\left(Q^{*}\right) \leq 0$. But if $Q^{*} \in \mathcal{A}$, then it cannot be a stationary state. Hence, if the trajectory reaches $Q^{*}$ in a finite time $t^{*}, \dot{P}\left(Q^{*}\right)<0$, so that, as it is easily checked, in a right neighborhood of $t^{*}, K(t)$ keeps decreasing, and so on, implying that such a trajectory, and the nearby ones, converge to $\hat{Q}$. In fact, in order to have a trajectory not converging to $\hat{Q}$, the above $Q^{*}$ had to be reached in infinite time. In other words, it had to be a stationary state and, precisely, a saddle. This completes the proof of the theorem.

Remark 5 It can be shown that in case $\varphi(0)>0\left(\right.$ or $\varphi(0)=0$ and $\varphi^{\prime}(0)>$ $0)$ no trajectory in $\mathcal{B}$ can tend, as $t \rightarrow+\infty$, to $K=0$.

Remark 6 Assume two interior stationary states exist, the saddle $Q^{*}$ and the $\operatorname{sink} \tilde{Q}, 0<K^{*}<\tilde{K}<\bar{N}$. Then it follows from straightforward computations that $\hat{P}<P^{*}<\tilde{P}$; that is, the pollution level $P$ in the boundary point $\hat{Q}$ is lower than in the internal stationary states, $Q^{*}$ and $\tilde{Q}$, when existing.

Note that the limit boundary point $\hat{Q}=(0, \bar{N}, \hat{P})$, where $\hat{P}$ is the solution of the equation $q \bar{N}^{\beta}-P(1+P)^{\gamma}=0$, coincides with the unique (globally attractive) stationary state of the one-sector dynamics that would be observed in the absence of the industrial sector. In this case, $K=0$ and $N=\bar{N}$ and the time evolution of $P$ would be described by the equation:

$$
\dot{P}=q \bar{N}^{\beta}(1+P)^{-\gamma}-P
$$

Along every trajectory of the three-dimensional system (8) approaching, as $t \rightarrow+\infty, \hat{Q}$, the economy tends (asymptotically) to become specialized in the resource-dependent sector.

Our next step will be to prove the following theorem.

Theorem 7 Let $\mathcal{C} \subseteq \mathcal{B}$ be a positively invariant region containing exactly one stationary state, that is, the $\operatorname{sink} \tilde{Q}=(\tilde{K}, \tilde{N}, \tilde{P})$. Then all the trajectories starting in $\mathcal{C}$ tend, as $t \rightarrow+\infty$, to $\tilde{Q}$.

${ }^{4}$ It can be checked that $\lim _{t \rightarrow T-} K(t)=0$ implies $T=+\infty$.

${ }^{5} Q^{*}$ can be considered the limit of a sequence of points $Q_{n}$ such that $\dot{K}\left(Q_{n}\right)>0$, $\dot{N}\left(Q_{n}\right)=0, \dot{P}\left(Q_{n}\right)<0$. 
In order to prove the above theorem, we need, first of all, to stretch the box where system (8) is studied. Precisely, we consider the box $\mathcal{B}^{\prime}=\{K, N \in$ $(0, \bar{N}), P \in(0,+\infty)\}$. Clearly $\mathcal{B}^{\prime} \supset \mathcal{B}$ and is positively invariant as well, with respect to (8). Hence we start by proving the following lemma.

Lemma 8 Consider system (8) defined in $\mathcal{B}^{\prime}$. Then there exist trajectories lying in $\mathcal{B}^{\prime}$ for all $t \leq 0$ and tending, as $t \rightarrow-\infty$, to the boundary point $Q_{\infty}=(\bar{N}, 0,+\infty)$.

PROOF. See Appendix A.2.

Now we can move to proving Theorem 7 . In fact, the full proof of the theorem is given in Appendix A.3. Here we just mention the main idea behind the proof. Assume, by contradiction, there exists in the region $\mathcal{C}$ described in the theorem's statement some $\omega$-limit set $\Sigma$ different from the $\operatorname{sink} \tilde{Q}$. Then $\Sigma$ is a compact set both positively and negatively invariant, whose trajectories are oscillating. Hence we reverse the time, i.e. we pose $\tau=-t$, and we show that we can choose $Q_{0}=\left(K_{0}, N_{0}, P_{0}\right) \in \Sigma$ such that, deriving with respect to $\tau$, $\dot{K}\left(Q_{0}\right)>0$ and $\dot{N}\left(Q_{0}\right)<0$. Hence the negative trajectory starting from $Q_{0}$, say $\alpha(\tau)$ with $\tau=-t$, should remain in $\mathcal{C}$ for all $\tau \in(0,+\infty)$. Vice-versa, we prove that, if $\alpha(\tau)$, with the above assumptions, remains in $\mathcal{B}^{\prime} \supset \mathcal{C}$ for all $\tau \in(0,+\infty)$, then $\lim _{\tau \rightarrow+\infty} \alpha(\tau)=Q_{\infty}=(\bar{N}, 0,+\infty)$, which doesn't even belong to the closure of $\mathcal{C}$, thus reaching a contradiction.

Remark 9 It follows from the proof of Theorem 7 that, if $\mathcal{C} \subset \mathcal{B}$, then the two-dimensional stable manifold of the saddle $Q^{*}$ is part of the boundary of $\mathcal{C}$.

The previous results are summarized in the following theorem.

Theorem 10 System (8), defined in the open box $\mathcal{B}$, can exhibit (generically) at most three regimes. Precisely:

(1) There exists a positively invariant region $\mathcal{A} \subseteq \mathcal{B}$ whose trajectories tend, as $t \rightarrow+\infty$, to the boundary point $\hat{Q}=(0, \bar{N}, \hat{P})$.

(2) There exists a positively invariant region $\mathcal{C} \subseteq \mathcal{B}$ whose trajectories tend, as $t \rightarrow+\infty$, to a sink $\tilde{Q}=(\tilde{K}, \tilde{N}, \tilde{P})$.

(3) There exists a positively invariant two-dimensional manifold $\mathcal{T} \subset \mathcal{B}$ whose trajectories tend, as $t \rightarrow+\infty$, to a saddle $Q^{*}=\left(K^{*}, N^{*}, P^{*}\right)$.

The numerical simulation in Fig. 1 illustrates the case in which only the regime 1 (of the above theorem) is observed in the box $\mathcal{B}$. Analogously, the numerical simulation in Fig. 2 illustrates the case in which only the regime 2 occurs in the box $\mathcal{B}$. Finally, the numerical simulation in Fig. 3 illustrates the case in which 


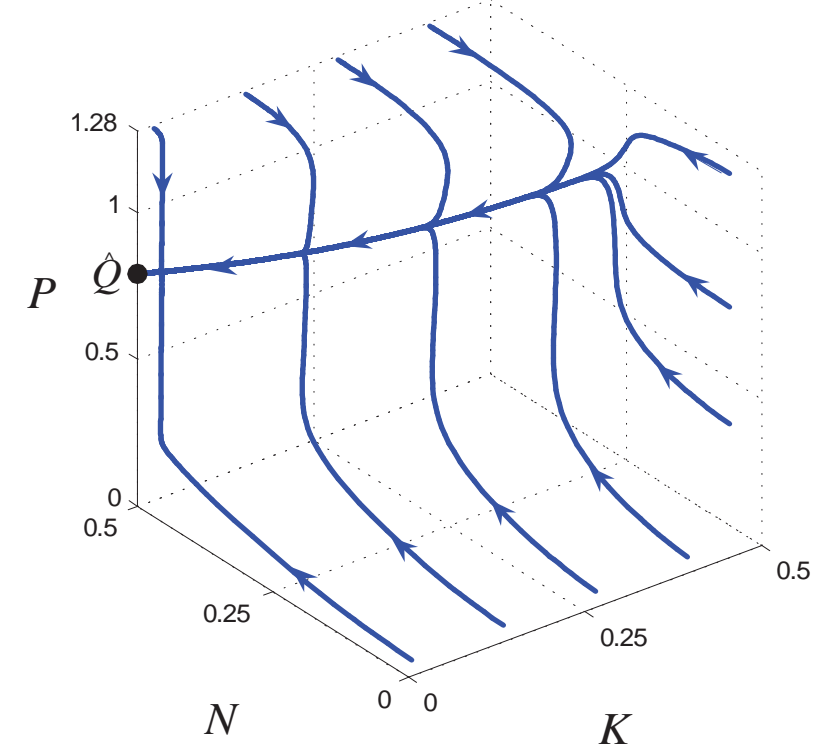

Fig. 1. Example of regime 1 (with $l=1, m=1, \beta=0.5, \gamma=0.5, \delta=0.06, \bar{N}=0.5$, $q=1.5>r=1.4$ such that $\varphi(0)=-0.25<0)$

all regimes take place in $\mathcal{B}$, depending on the initial values of the state variables $K, N$, and $P$. In the case of Fig. 3, the two-dimensional stable manifold of the saddle $Q^{*}$ (along which regime 3 occurs) separates the trajectories of regime 1 from those of regime 2. The phase portraits illustrated in Figs 1-3 represent the only ones that can be generically observed in the box $\mathcal{B}$ under the dynamic system (8).

Remark 11 It follows from the above theorems that a region $\mathcal{A}$ whose trajectories tend to the boundary point $\hat{Q}^{6}$ exists if and only if $\varphi(0)<0$ or $\varphi(0)=0$ and $\varphi^{\prime}(0) \leq 0$. In the former case, we have $\varphi(0)=1+q \bar{N}-\bar{N}^{\frac{\beta-1}{\gamma}}<0$, which implies, as it is easily checked, $\hat{P}>q \bar{N}$, where $\hat{P}$ is the solution of the equation $q \bar{N}^{\beta}-P(1+P)^{\gamma}=0$. Hence, replacing in the previous equation the first $P$ by $q \bar{N}$, we get $\bar{N}^{\beta-1}-(1+\hat{P})^{\gamma}>0$, i.e. $\bar{N}^{\beta-1} /(1+\hat{P})^{\gamma}>1$. In other words, at the boundary attractor $\hat{Q}$, the revenues of the workers employed in the F-sector are higher than at the possible sink, where the wage rate is, by the equilibrium conditions, equal to 1 . Vice-versa, when $\hat{Q}$ is not attracting, which implies $\varphi(0) \geq 0$, we get $\bar{N}^{\beta-1} /(1+\hat{P})^{\gamma} \leq 1$ (the strict inequality holding if $\varphi(0)>0)$.

6 Remember that such point corresponds to the unique (globally attractive) stationary state of the one-sector dynamics (12) that would be observed in absence of the industrial sector. 


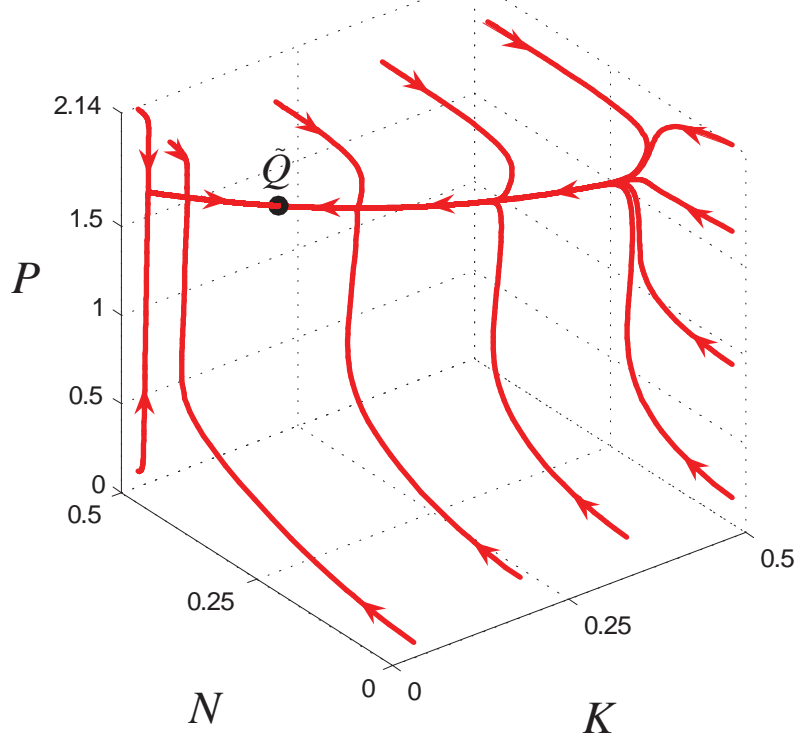

Fig. 2. Example of regime 2 (with $l=1, m=1, \beta=0.5, \gamma=0.5, \delta=0.06, \bar{N}=0.5$, $q=4>r=1.4$ such that $\varphi(0)=1>0)$

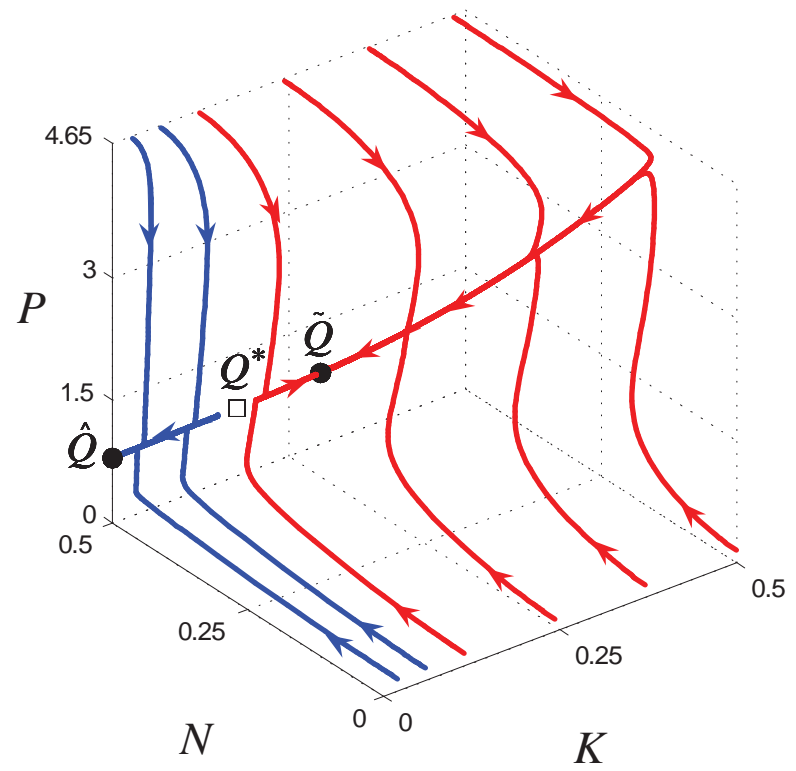

Fig. 3. Example of regime 3 (with $l=1, m=1, \beta=0.5, \gamma=0.5, \delta=0.06, \bar{N}=0.5$, $q=1.5<r=9$ such that $\varphi(0)=-0.25<0)$ 
It follows from the above theorem that the three possible dynamic regimes of system (A.2) coexist if and only if the system exhibits two stationary states in $\mathcal{B}$, i.e. the saddle $Q^{*}$ and the sink $\tilde{Q}$. In this case, the stable manifold of $Q^{*}$ separates the basins of attraction of $\tilde{Q}$ and the boundary attractor $\hat{Q}$. Then, it becomes interesting to know more about the shape of such stable manifold. To this end we prove the following

Theorem 12 Assume system (A.2) has two stationary states in $\mathcal{B}$, a saddle $Q^{*}=\left(K^{*}, N^{*}, P^{*}\right)$ and a sink $\tilde{Q}=(\tilde{K}, \tilde{N}, \tilde{P})$ : then there exists a region $\mathcal{S}$ in the plane $(K, N), \mathcal{S}=\left\{K_{1}<K<K_{2}, N_{1}(K)<N<N_{2}(K)\right\}$, having the following properties.

(1) $K_{1}<K^{*}<K_{2} \leq \tilde{K}, N_{1}(K)<\bar{N}-K<N_{2}(K)$.

(2) For each $\left(K_{0}, N_{0}\right) \in \mathcal{S}$, there exists exactly one value $P_{0}^{T}$, i.e. $P_{0}^{T}=$ $\varphi\left(K_{0}, N_{0}\right)$, such that: a trajectory starting from $\left(K_{0}, N_{0}, P_{0}\right)$, with $P_{0}<$ $P_{0}^{T}$, tends to the boundary point $\hat{Q} ;$ a trajectory starting from $\left(K_{0}, N_{0}, P_{0}\right)$, with $P_{0}>P_{0}^{T}$, tends to the sink $\tilde{Q}$; the trajectory starting from $\left(K_{0}, N_{0}, P_{0}^{T}\right)$ tends to the saddle $Q^{*}$.

PROOF. Let us consider the plane $\pi$ of equation $N+K=\bar{N}$, intersecting the box $\mathcal{B}$ in a rectangle $\mathcal{R}$, where we choose $P$ and $N$ as coordinates, so that $\mathcal{R}=(0, \bar{P}) \times(0, \bar{N})$. It follows from the proof of Theorem 4 that, when $N$ is sufficiently high (hence $K$ is sufficiently low), the trajectories from the corresponding strip of $\mathcal{R}$ tend to $\hat{Q}$. Hence the intersection of $\mathcal{R}$ with the stable manifold of $Q^{*}$, which separates the above two regimes, is a curve $\Gamma$ contained in a strip $\left\{N_{1}^{\prime}<N<N_{2}^{\prime} / 0<N_{1}^{\prime}<N^{*}<N_{2}^{\prime}\right\}$. Moreover, it follows again from the proof of Theorem 4 that, when $N_{0}>N^{*}$, a trajectory from $\left(\bar{N}-N_{0}, N_{0}, P_{0}\right)$, with $P_{0} \leq N_{0}^{\frac{\beta-1}{\gamma}}-1$, tends to $\hat{Q}$. On the other hand, it is easily computed that for $N_{0}>N^{*}, K_{0}=\bar{N}-N_{0}, P_{0}>N_{0}^{\frac{\beta-1}{\gamma}}-1, \dot{N}\left(K_{0}, N_{0}, P_{0}\right)$ and $\dot{P}\left(K_{0}, N_{0}, P_{0}\right)$ are $<0$, while for $\tilde{N}<N_{0}<N^{*}, K_{0}=\bar{N}-N_{0}, P_{0}<$ $N_{0}^{\frac{\beta-1}{\gamma}}-1, \dot{N}\left(K_{0}, N_{0}, P_{0}\right)$ and $\dot{P}\left(K_{0}, N_{0}, P_{0}\right)$ are $>0$.

Suppose, now, that, near $Q^{*}, \Gamma$ is the graph of a decreasing function $N(P)$, so that $\frac{d N}{d P}<0$ as $P$ lies in a left neighborhood of $P^{*}$. Consider a closed tract $\Gamma^{\prime}$ of $\Gamma$ where that occurs, $Q^{*} \notin \Gamma^{\prime}$. Pose $H=N+K$ and take $Q_{0}=$ $\left(H_{0}, N_{0}, P_{0}\right) \in \Gamma^{\prime}$ (hence $\left.H_{0}=\bar{N}\right)$. To fix the ideas, we can assume $Q_{0}$ is the end-point of $\Gamma^{\prime}$ with the higher $N$. Recalling that on $\pi \dot{K}=0$ and therefore $\dot{N}<0$ implies $\dot{H}<0$, consider a sufficiently small box $\mathcal{B}^{\prime}=\left[H_{0}-a, H_{0}\right] \times$ $\left[N_{0}-b, N_{0}\right] \times\left[P_{0}, P_{0}+c\right], a, b, c>0$. The intersection of the side $\left\{P=P_{0}\right\}$ of $\mathcal{B}^{\prime}$ with the stable manifold of $Q^{*}, \mathcal{T}$, is a curve $\alpha$ which can be parametrized by $N \in\left[N_{0}-b, N_{0}\right]$. In fact, being $T$ invariant (i.e., constituted by trajectories), along such a curve $\alpha \frac{d H}{d N}=\frac{\dot{H}\left(H, N, P_{0}\right)}{\dot{N}\left(H, N, P_{0}\right)}$. Moreover, if $B^{\prime}$ is small enough, there 
exist $r_{1}>r_{2}>0$ such that, in $B^{\prime},-r_{1} \leq \dot{N}, P \leq-r_{2}$. Take now $Q_{0}^{\prime}=$ $\left(H_{0}, N_{0}, P_{0}^{\prime}\right)$, with $P_{0}^{\prime}=P_{0}+\delta, \delta>0$ being sufficiently small (so that $Q_{0}^{\prime}$ belongs to the basin of $\tilde{Q}$ ). Then the trajectory from $Q_{0}^{\prime}$ stays for a certain time in $B^{\prime}$, and in fact we can parametrize it too by $N$, so that it is represented by a curve $\gamma(N)=(H(N), N, P(N)), N_{0}-b \leq N \leq N_{0}$. Then, along $\gamma(N)$, $\frac{d P}{d N} \geq \frac{r_{2}}{r_{1}}>0$. Moreover, being $\dot{N}<0$, it is easily computed that, for given $H$ and $N, \frac{d H}{d N}=1+\frac{\dot{K}}{\dot{N}}$ is higher for a higher $P$. So, if $\delta$ is sufficiently small, the trajectory $\gamma$ will reach the side $\left\{P=P_{0}\right\}$ of $B^{\prime}$ for some pair $\left(H_{1}, N_{1}\right) \in$ $\left(H_{0}-a, H_{0}\right) \times\left(N_{0}-b, N_{0}\right)$. Should the trajectory remain "to the right" of $T$, then, for what we have noticed, the corresponding pair $\left(H_{1}, \hat{N}_{1}\right)$ on $\alpha=$ $T \cap\left\{P=P_{0}\right\}$ would satisfy $\hat{N}_{1}>N_{1}$. It follows that, for our choice of a sufficiently small $B^{\prime}$, we would have a point $\left(H_{1}, N_{1}, P_{1}\right)$ on $T$ with $P_{1}>P_{0}$, leading to a contradiction, since we have supposed that $\gamma$ lies "to the right" of $T$. Hence $\gamma$ reaches the side $\left\{P=P_{0}\right\}$ of $B^{\prime}$ by intersecting the invariant manifold $T$, which again leads to a contradiction.

It follows that, for $P$ lying in a right neighborhood of $P^{*}$, the points in $\mathcal{R}$ of the curve $C=\left\{N=(1+P)^{\frac{\gamma}{\beta-1}}, N \geq \tilde{N}\right\}$ (which is the graph of a decreasing function) lie to the right of $\Gamma$, and thus belong to the basin of attraction of $\tilde{Q}$. If it happened that $\Gamma$ crossed again the curve $C$ for some $P>P^{*}$, then there should exist, as it is easily observed, a tract of $\Gamma$ where $\frac{d N}{d P}<0$ with $P>P^{*}$. Again, by an argument analogous to the previous one, we are led to a contradiction, considering the trajectory from a point $Q_{0}^{\prime}$ sufficiently close to this tract and lying to the left of $\Gamma$ (so that $\dot{N}\left(Q_{0}^{\prime}\right), \dot{P}\left(Q_{0}^{\prime}\right)>0$ ). Hence the curve $C$, for $P>P^{*}$, belongs to the basin of $\tilde{Q}$.

Suppose, now, that there exists a tract $\Gamma^{\prime}$ of $\Gamma$ such that, for $P \in\left[P_{1}, P_{2}\right]$, $P_{1}>P^{*}, \Gamma^{\prime}$ is the graph of a function $N(P)$ satisfying $\frac{d N}{d P}<0 .{ }^{7}$ Hence, along $\Gamma^{\prime}, \dot{N}, \dot{P}<0$ and, as above, $-r_{1} \leq \dot{N}, P \leq-r_{2}$ for suitable $r_{1}>r_{2}>0$. Then we can consider a point $Q_{0}^{\prime}$ sufficiently close to $\Gamma^{\prime}$ lying to the right of $\Gamma$ (i.e., for our assumption, in the basin of attraction of $\hat{Q})$. Again, it follows that the trajectory from $Q_{0}^{\prime}$ would reach the stable manifold of $Q^{*}$ within a finite time, leading to a contradiction.

The same argument can be can be applied to rule out the existence of a tract $\Gamma^{\prime}$ of $\Gamma$ where $\frac{d N}{d P}<0$ as $P<P^{*}$ and $\tilde{N}<N<N^{*}$ (in this case $Q_{0}^{\prime}$ can be chosen as lying to the left of $\Gamma^{\prime}$, so that $\left.\dot{N}\left(Q_{0}^{\prime}\right), \dot{P}\left(Q_{0}^{\prime}\right)>0\right)$.

In conclusion, $\Gamma$ is the graph of an increasing function $N(P)$, hence $P(N)$, in a strip $\left(N_{1}, N_{2}\right) \subset \mathcal{R}$, where $N_{1} \leq \tilde{N}<N^{*}<N_{2}$, which implies that for

7 Observe that, since $q<r$ (as the existence of two stationary states implies), the curve $\{\dot{P}=0\} \cap \mathcal{R}$ is represented, near $Q^{*}$, by the graph of a function $N(P)$ such that $\frac{d N}{d P}\left(P^{*}\right)<0$. 
(a)

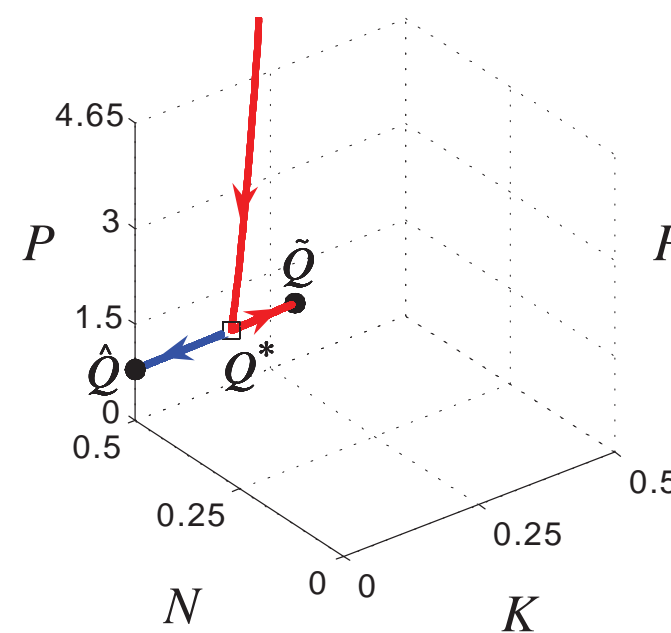

(b)

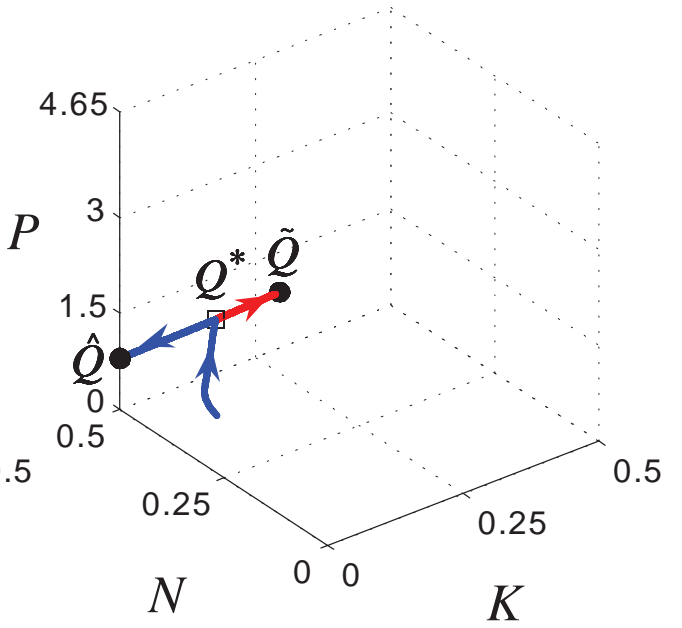

Fig. 4. Two examples illustrating Theorem 12: $(a)$ the blue trajectory starts from $\left(K_{0}, N_{0}, P_{0}\right)=\left(K^{*}-0.01, \bar{N}-K_{0}, N_{0}^{-1}-1\right)$, whereas the red trajectory from $\left(K_{0}, N_{0}, 28\right)$; $(b)$ the red trajectory starts from $\left(K_{0}^{\prime}, N_{0}^{\prime}, P_{0}^{\prime}\right)=\left(K^{*}+0.01, \bar{N}-K_{0}^{\prime}, N_{0}^{\prime-1}-1\right)$, whereas the blue trajectory from $\left(K_{0}^{\prime}, N_{0}^{\prime}, 0\right)$

$(K, N)$ belonging to a suitable region $\mathcal{S}$, as the one described in the statement of the theorem, the stable manifold of $Q^{*}$ can be represented as the graph of a function $P(K, N)$.

Fig. 4 shows trajectories starting from the same initial values of $K$ and $N$, but different initial values of $P$, approaching either the boundary point $\hat{Q}$ or the $\operatorname{sink} \tilde{Q}$.

Remark 13 The above theorem implies that in a neighborhood of $Q^{*}$ the stable manifold of the saddle can be interpreted as the graph of a function $P=\varphi(K, N)$. Moreover, it follows from the proof of the theorem that such a manifold, in a neighborhood of $Q^{*}$, can also be seen as the graph of a function $N=\psi(K, P)$. Hence, if $\left(K_{0}, P_{0}\right)$ is sufficiently close to $\left(K^{*}, P^{*}\right)$, there exist a $\delta>0$ and a function $N_{0}^{T}=\psi\left(K_{0}, P_{0}\right)$ such that: a trajectory starting from $\left(K_{0}, N_{0}, P_{0}\right)$, with $N_{0}^{T}<N_{0}<N_{0}^{T}+\delta$, tends to the boundary point $\hat{Q}$; a trajectory starting from $\left(K_{0}, N_{0}, P_{0}\right)$, with $N_{0}^{T}-\delta<N_{0}<N_{0}^{T}$, tends to the sink $\tilde{Q}$; the trajectory starting from $\left(K_{0}, N_{0}^{T}, P_{0}\right)$ tends to the saddle $Q^{*}$. 


\section{Interpretation of the main results and concluding remarks}

In this paper we have analyzed the dynamics of a small open economy with two sectors (a farming sector and an industrial sector), free inter-sectoral labor mobility and heterogeneous agents (farmers and industrial entrepreneurs). We studied the dynamics of the three crucial variables of the model ( $N, K$ and $P$ ) and gave a complete classification of the dynamic regimes that can be observed. More specifically, we showed that the three-dimensional dynamic system of the model, defined (after a suitable rescaling) in a positively invariant box $\mathcal{B}=\{(K, N, P) \in(0, \bar{K}) \times(0, \bar{N}) \times(0, \bar{P})\}$, can (generically) exhibit at most three regimes. More precisely:

(1) There exists a positively invariant region $\mathcal{A} \subseteq \mathcal{B}$ whose trajectories tend, as $t \rightarrow+\infty$, to the boundary point $\hat{Q}=(0, \bar{N}, \hat{P})$, where $K=0$ and therefore the economy "specializes" in the production of the F-sector.

(2) There exists a positively invariant region $\mathcal{C} \subseteq \mathcal{B}$ whose trajectories tend, as $t \rightarrow+\infty$, to a sink $\tilde{Q}=(\tilde{K}, \tilde{N}, \tilde{P})$, with $\tilde{K}>0$ and $\tilde{N} \in(0, \bar{N})$, where the two sectors coexist.

(3) There exists a positively invariant two-dimensional manifold $\mathcal{T} \subset \mathcal{B}$ whose trajectories tend, as $t \rightarrow+\infty$, to a saddle $Q^{*}=\left(K^{*}, N^{*}, P^{*}\right)$, with $K^{*}>0$ and $N^{*} \in(0, \bar{N})$.

We showed (Theorem 1) that the presence and possible coexistence of the three regimes depend on the features of a function $\varphi(K)=q(\bar{N}-K)+$ $r K-(\bar{N}-K)^{\frac{\beta-1}{\gamma}}+1$.

In particular, regimes 1, 2, and 3 exist simultaneously if and only if: $a) \varphi(0)=$ $\left.q \bar{N}-\bar{N}^{\frac{\beta-1}{\gamma}}+1<0 ; b\right) \varphi(K)$ has exactly one maximum $\left.K_{M} \in(0, \bar{N}) ; c\right)$ $\varphi\left(K_{M}\right)>0$.

This implies $q<r$, i.e., that the environmental impact of the F-sector is lower, after a suitable rescaling, than the environmental impact of the I-sector, and that the rescaled population size $\bar{N}$ is low enough, as $1>(q \bar{N}+1) \bar{N}^{\frac{1-\beta}{\gamma}}$. When this is the case, the two attractors $\tilde{Q}$ and $\hat{Q}$ coexist and their basins of attraction are separated by the two-dimensional stable manifold of the saddle $Q^{*}$. Furthermore, we noticed that:

(1) The boundary point $\hat{Q}$ coincides with the unique (globally attractive) stationary state of the one-sector dynamics (12) that would be observed in absence of the industrial sector (in such an economy, $K=0$ and $N=\bar{N}$ always hold). Along every trajectory of the three-dimensional system (8) approaching $\hat{Q}$, the economy tends (asymptotically) to become specialized in the resource-dependent sector. 
(2) When the two interior stationary states $Q^{*}$ and $\tilde{Q}$ exist, then $0<K^{*}<$ $\tilde{K}<\bar{N}$ and $\hat{P}<P^{*}<\tilde{P}$ hold (see Remark 6), where $\hat{P}$ is the pollution level $P$ evaluated at the boundary point $\hat{Q}$.

(3) If the boundary point $\hat{Q}$ is attractive, then in such state the revenues of the workers employed in the F-sector are higher than in the sink $\tilde{Q}$. The opposite holds when the boundary point $\hat{Q}$ is not attractive (see Remark 11). In the former context, we can say that a perverse structural change occurs (in the sense of Lopez, 2007; see the Introduction of this paper) when the economy converges to the $\operatorname{sink} \tilde{Q}$, where both sectors coexist. The opposite holds when the boundary point $\hat{Q}$ is not attractive (see Remark 11).

According to Theorem 12, when two interior stationary states exist, the initial pollution level $P(0)$ may play a crucial role, given the initial values $K(0)$ and $N(0)$ of the other two variables, in determining equilibrium selection. In fact, if $K(0)$ and $N(0)$ satisfy the conditions of the theorem, then there exists a threshold value $P^{T}$ such that, starting from $(K(0), N(0), P(0))$, the economy converges to $\hat{Q}$ (where it becomes specialized in the F-sector) if $P(0)<P^{T}$, while it converges to $\tilde{Q}$ (where the economy gets industrialized) if $P(0)>P^{T}$. A higher initial level $P(0)$ of pollution implies lower labor productivity in the F-sector; therefore, as in the seminal work by Matsuyama (1992), in our model low productivity of labor in the resource-dependent sector is the engine of the industrialization process. However, the self-re-enforcing process of industrialization driving the economy towards the stationary state $\tilde{Q}$ is always associated with a decrease in workers' revenues (in the context in which the boundary point $\hat{Q}$ is attractive), differently from Matsuyama's work.

Analogously, also the initial value $N(0)$ of $N$ may play a role in equilibrium selection, as pointed out in Remark 13. So, in the case in which the boundary point $\hat{Q}$ is attractive, the convergence to the interior sink from a point close to the saddle $Q^{*}$ can be considered as the consequence of a coordination failure of workers.

Clearly, our result on workers' revenues is strictly linked to our assumption that the industrialization process generates negative environmental externalities, but not positive ones, as is assumed, on the contrary, by Matsuyama. In the case in which both types of externalities condition the dynamics of the economy, it may happen that negative externalities - through the mechanism analyzed in our paper - lead economic agents towards a better exploitation of positive externalities. Obviously, in such a context, the effect of positive externalities may counterbalance the effect of negative externalities.

Although our conceptual framework is too simple fully to catch all dynamic aspects of the growth paths of developing countries, we believe that some of the 
latter are consistent with the narratives behind the model we have proposed and this encourages further research along the lines suggested in this paper.

\section{A Appendix}

\section{A.1 Proof of Theorem 1}

Let $Q_{0}=\left(K_{0}, N_{0}, P_{0}\right) \in \mathcal{B}$ be a stationary state of system $(A .2)$. Then it is easily computed that $\varphi\left(K_{0}\right)=0$. Moreover, $\lim _{K \rightarrow \bar{N}} \varphi(K)=-\infty$ and $\varphi^{\prime \prime}(K)<0$ as $K \in(0, \bar{N})$. It follows that the stationary states in $\mathcal{B}$ are at most two, according to the conditions stated in the theorem. Furthermore, the Jacobian matrix $J\left(Q_{0}\right)$ is given by

$$
J\left(Q_{0}\right)=\left(\begin{array}{lll}
-l \delta & -l \delta & 0 \\
\frac{-m(1-\delta)}{K_{0}} & \frac{-m(1-\delta)}{K_{0}}-\frac{m(1-\beta)}{N_{0}} & \frac{-m \gamma}{1+P_{0}} \\
r(1-\delta) & -r \delta+q \beta & \frac{-q \gamma N_{0}}{1+P_{0}}-1
\end{array}\right)
$$

It follows, by easy computations, that $\operatorname{sign}\left[\operatorname{det}\left(J\left(Q_{0}\right)\right)\right]=\operatorname{sign}\left[\varphi^{\prime}\left(K_{0}\right)\right]$ and $\operatorname{tr}\left(J\left(Q_{0}\right)\right)<0$. Therefore, if $\varphi^{\prime}\left(K_{0}\right)>0, Q_{0}$ is a saddle with a two-dimensional stable manifold. If, instead, $\varphi^{\prime}\left(K_{0}\right)<0$, then the characteristic polynomial of $J\left(Q_{0}\right)$ is given by

$$
-\lambda^{3}+\operatorname{tr}(J) \lambda^{2}-\sigma(J) \lambda+\operatorname{det}(J)
$$

and it is easily calculated that

$$
|\operatorname{tr}(J)| \cdot \sigma(J)>|\operatorname{det}(J)|
$$

Therefore, the Routh-Hurwicz conditions yield that $Q_{0}$ is a sink. This completes the proof of the theorem.

\section{A.2 Proof of Lemma 8}

We consider the change of time $\tau=-t$ and continue to denote the derivatives with respect to $\tau$ by $\dot{K}, \dot{N}, \dot{P}$. Hence the system, to be studied in $\mathcal{B}^{\prime}$, becomes (A.2). 
In order to simplify the notations we can pose $P^{\prime}=P+1$, renaming $P^{\prime}$ as $P$. Then, writing $\dot{P}=P-f(K, N, P), \dot{P}>\frac{1}{2} P$ if $P \geq P_{0}$ is sufficiently large and it is easily checked that $P \rightarrow+\infty$ iff $\tau \rightarrow+\infty$. Therefore for $P \geq P_{0}$ we can replace $\tau$ by $P$ and consider, in the plane $(K, N)$, the non-autonomous system

$$
\begin{aligned}
& \frac{d K}{d P}=\frac{l K^{1-\delta}\left[K^{\delta}-(\bar{N}-N)^{\delta}\right]}{P-f(K, N, P)} \\
& \frac{d N}{d P}=\frac{m\left[(\bar{N}-N)^{\delta-1} K^{1-\delta}-N^{\beta-1} P^{-\gamma}\right]}{P-f(K, N, P)}
\end{aligned}
$$

Now, set $K=\bar{N}-H$, we consider, for each $P \geq P_{0}$, the trajectories starting, respectively, at $Q_{1}(P)=(H, N, P)$ satisfying $N=P^{-\frac{\gamma}{1-\beta}}-a P^{-\frac{2 \gamma}{1-\beta}}, \frac{d N}{d P}=0$ and at $Q_{2}(P)=(H, N, P)$ satisfying $H=N=P^{-\frac{\gamma}{1-\beta}}-a P^{-\frac{2 \gamma}{1-\beta}}$, with $a>0$ being sufficiently small.

Hence the former trajectories start from a minimum value of $N$ and the latter ones from a minimum value of $H$. In the plane $(H, N)$ the points $Q_{1}(P)$ fill a curve $C_{1}$, the points $Q_{2}(P)$ a curve $C_{2}$ (in fact, a segment on the line $N=H)$, which, together with the segment $C_{3}=\left[Q_{1}^{0}, Q_{2}^{0}\right]$ lying on $\left\{N=N_{0}=P_{0}^{-\frac{\gamma}{1-\beta}}-a P_{0}^{-\frac{2 \gamma}{1-\beta}}\right\}$, are the sides of a curvilinear triangle $\mathcal{T}$. Now, it is easily checked that a trajectory starting, at $P=P_{0}$, from a point of $C_{3}$ may reach in $T$ at most a minimum (and not a maximum) value of $N$ (while in $\left.T-C_{2} \frac{d H}{d P}<0\right)$. In fact, $\frac{d N}{d P}=0$ corresponds to $\dot{N}=0$ in system $(A .2)$, so that $\ddot{N}=\frac{\dot{K}}{(\bar{N}-N)^{1-\delta} K^{\delta}}+\gamma \frac{\dot{P}}{N^{1-\beta} P^{1+\gamma}}>0$, as $\dot{K}$ and $\dot{P}$ are both positive inside $\mathcal{T}$. Moreover, it follows from the continuous dependence on initial conditions that trajectories starting at $P=P_{0}$ from a suitable right neighborhood of $Q_{1}^{0}$ on $C_{3}$ cross the side $C_{1}$ of $\mathcal{T}$, while trajectories starting at $P=P_{0}$ from a suitable left neighborhood of $Q_{2}^{0}$ on $C_{3}$ cross the side $C_{2}$. In fact, the same argument yields, as it is easily seen, that all the points both of $C_{1}$ and $C_{2}$ are reached by trajectories starting at $P=P_{0}$ from $C_{3}$. Therefore there exists some trajectory $\Gamma$, lying in $\mathcal{T}$, starting from $C_{3}$ at $P=P_{0}$ and tending to $H=N=0$. Then along $\Gamma \frac{d H}{d P}<0$ (i.e., $\frac{d K}{d P}>0$ ) and $\frac{d N}{d P}<0$, implying $N^{\beta-1} P^{-\gamma}>(\bar{N}-N)^{\delta-1} K^{1-\delta}>1$, that is $H<N<P^{-\frac{\gamma}{1-\beta}}$. It follows that $H(P)$ decreases not faster than $P^{-\frac{\gamma}{1-\beta}}$ and thus $H \rightarrow 0$ implies $P \rightarrow+\infty$.

This concludes the proof of the Lemma.

\section{A.3 Proof of Theorem 7}

We will proceed by contradiction. Recalling Remark 5, assume there exists in $\mathcal{C}$ some $\omega$-limit set $\Sigma$ different from the sink $\tilde{Q}$. Then every trajectory $\Gamma \subseteq \Sigma$ stays in $\mathcal{C}$ and therefore in $\mathcal{B}^{\prime}$ for all $t \in(-\infty,+\infty)$ and moreover is 
oscillating (i.e., along $\Gamma, K(t), N(t), P(t)$ reach relative minima and maxima at infinitely many positive and negative times). Hence, consider the change of time $\tau=-t$ and, continuing to denote the derivatives with respect to $\tau$ by $\dot{K}, \dot{N}, \dot{P}$, the system:

$$
\begin{aligned}
\dot{K} & =l K^{1-\delta}\left[K^{\delta}-(\bar{N}-N)^{\delta}\right] \\
\dot{N} & =m\left[(\bar{N}-N)^{\delta-1} K^{1-\delta}-N^{\beta-1}(1+P)^{-\gamma}\right] \\
\dot{P} & =P-q N^{\beta}(1+P)^{-\gamma}-r(\bar{N}-N)^{\delta} K^{1-\delta}
\end{aligned}
$$

defined in $\mathcal{B}^{\prime}$. Along a trajectory $\alpha(\tau)$ lying in $\mathcal{B}^{\prime}$ for all $\tau \geq 0$ and oscillating, $K(\tau)$ will have a minimum, say, at $\tau_{1}$, implying $\dot{N}\left(\tau_{1}\right) \geq 0$ and $\dot{N}(\tau)>0$ in a right neighborhood of $\tau_{1}$. Then there will exist $\tau_{2}>\tau_{1}$, where $N(\tau)$ has a maximum. Since $\dot{K}\left(\tau_{2}\right)>0$, this is easily seen to imply $\dot{P}\left(\tau_{2}\right)<0$. Let us set $\tau_{2}=0$ and examine $\alpha(\tau)$ for $\tau>0$. As in the proof of Theorem 4, we can consider, for any $K^{*} \in(0, \bar{N})$, two curves on the plane $K=K^{*}$, i.e., the graph of a decreasing function $N=\varphi^{*}(P)$ corresponding to $\dot{N}=0$ (with $\varphi^{*}(-1)=$ $\left.\bar{N}, \lim _{P \rightarrow+\infty} \varphi^{*}(P)=0\right)$ and the graph of a function $P=\psi^{*}(N)$ exhibiting exactly one maximum as $N \in(0, \bar{N})$ and such that $\psi^{*}(0)=r \bar{N}^{\delta}\left(K^{*}\right)^{1-\delta}$, $\psi^{*}(\bar{N})=\hat{P}$ (satisfying $\left.\hat{P}(1+\hat{P})^{\gamma}=q \bar{N}^{\beta}\right)$. Moreover, in the positive quadrant of the plane $K=K^{*}, \dot{N}>0$ at points $(P, N)$ such that $N>\varphi^{*}(P)$ and $\dot{P}$ $>0$ at points $(P, N)$ such that $P>\psi^{*}(N)$. Finally, we observe that $\dot{K}>0$ at points where $N>\bar{N}-K^{*}$, while the intersection between $\dot{K}=0$ and $\dot{N}=0$ takes place at $R^{*}=\left(\bar{N}-K^{*},\left(\bar{N}-K^{*}\right)^{-\frac{1-\beta}{\gamma}}-1\right)$.

Therefore, take $K^{*}$ sufficiently close to $\bar{N}$, say $0<\bar{N}-K^{*}<\varepsilon$, $\varepsilon$ being sufficiently small. Consider the previous trajectory $\alpha(\tau)$ and recall we assumed $\dot{K}(\tau)>0$ and $\dot{N}(\tau)<0$ in a right neighborhood of $\tau=0$ (in fact, we can assume $\dot{K}(0)>0$ and $\dot{N}(0)<0)$. Set $\alpha(0)=\left(K_{0}, N_{0}, P_{0}\right)$ and suppose $\bar{N}-K_{0}<\varepsilon$. If $\varepsilon>0$ is sufficiently small, then $P_{0}^{*}=\left(\bar{N}-K_{0}\right)^{-\frac{1-\beta}{\gamma}}-1$ is such that, for $P \geq P_{0}^{*}, \dot{P}>\frac{1}{2} P$, implying, as it is easily checked, that along the trajectory starting at $Q_{0}^{*}=\left(K_{0}, \bar{N}-K_{0}, P_{0}^{*}\right) P(\tau) \rightarrow+\infty$ as $\tau \rightarrow+\infty$. First of all, we prove that in this case, if $\alpha(\tau)$ remains in $\mathcal{B}^{\prime}$ for all $\tau \geq 0$, then it cannot cross at any positive time $\tau$ a line $\left\{K=K^{*}, N=\bar{N}-K^{*}\right\}, K^{*}>K_{0}$. In fact, assume that to be the case, i.e., $K\left(\tau_{1}\right)=\bar{N}-N\left(\tau_{1}\right)$ at some $\tau_{1}>0$ with $K_{1}>K_{0}$. We observe that along the trajectory starting from $Q_{1}^{*}=$ $\left(K_{1}, \bar{N}-K_{1},\left(\bar{N}-K_{1}\right)^{-\frac{1-\beta}{\gamma}}-1\right) P$ increases, as $P_{1}^{*}=\left(\bar{N}-K_{1}\right)^{-\frac{1-\beta}{\gamma}}-1>$ $P_{0}^{*}$, and so does $K$ (in fact, $\dot{K}(0)=\ddot{K}(0)=0$, but $\dddot{K}(0)>0$ ). Therefore, by the continuous dependence on initial conditions, along trajectories leaving from the line $\left\{K=K_{1}, N=\bar{N}-K_{1}\right\}$ at points lying in a suitable left 
neighborhood of $Q_{1}^{*}, K$ and $N$ will be also increasing after some time $\bar{\tau}>0$. Now, let us go back to our trajectory $\alpha(\tau)^{8}$, which will intersect $\dot{N}=0$ at a point $Q_{2}=\left(K_{2}, N_{2}, P_{2}\right)$, such that $\left(1+P_{2}\right)^{\gamma}=N_{2}^{\beta-1}\left(\frac{\bar{N}-N_{2}}{K_{2}}\right)^{1-\delta}>N_{2}^{\beta-1}>$ $\left(\bar{N}-K_{1}\right)^{\beta-1}$ (since $\dot{K}<0$ implies $\left.K<\bar{N}-N\right)$. If $\alpha(\tau)$ should not cross again $\dot{K}=0$, i.e. the plane $K=\bar{N}-N$, then, following the previous considerations, there should be a trajectory starting from the line $\left\{K=K_{1}, N=\bar{N}-K_{1}\right\}$ tangent to a line $\left\{K=K_{3}, N=\bar{N}-K_{3}\right\}$ at a point $Q_{3}=\left(K_{3}, \bar{N}-K_{3}, P_{3}\right)$ with $K_{3}<K_{1}$. However, $\frac{d N}{d P}\left(Q_{3}\right)=0$ is equivalent to $\dot{N}\left(Q_{3}\right)=0$, so that $P_{3}=\left(\bar{N}-K_{3}\right)^{-\frac{1-\beta}{\gamma}}-1$ On the other hand that trajectory would have previously crossed $\dot{N}=0$ at a point $\tilde{Q}_{2}=\left(\tilde{K}_{2}, \tilde{N}_{2}, \tilde{P}_{2}\right)$, such that, as above, $\left(1+\tilde{P}_{2}\right)^{\gamma}=\tilde{N}_{2}^{\beta-1}\left(\frac{\bar{N}-\tilde{N}_{2}}{\tilde{K}_{2}}\right)^{1-\delta}>\tilde{N}_{2}^{\beta-1}$, implying that $P$ increases between $\tilde{Q}_{2}$ and $Q_{3}$. But $\left(1+P_{3}\right)^{\gamma}=N_{3}^{\beta-1}<\tilde{N}_{2}^{\beta-1}<\left(1+\tilde{P}_{2}\right)^{\gamma}$, leading to a contradiction. It follows that $\alpha(\tau)$ must cross again $\dot{K}=0$ at a time, say, $\bar{\tau}>\tau_{1}$. Consequently, as $P(\tau) \rightarrow+\infty, \dot{N}(\tau)>c>0$ for $\tau>\bar{\tau}$, implying that $N(\tau)$ reaches the value $\bar{N}$ within a finite time, contradicting the assumption that $\alpha(\tau)$ remains in $\mathcal{B}^{\prime}$ for all $\tau \geq 0$. Hence, along $\alpha(\tau), K(\tau)$ keeps increasing. It follows: $\lim _{\tau \rightarrow+\infty} K(\tau)=\bar{N}$ and $\lim _{\tau \rightarrow+\infty} \dot{K}(\tau)=0$, implying $\lim _{\tau \rightarrow+\infty}$ $N(\tau)=\lim _{\tau \rightarrow+\infty} \dot{N}(\tau)=0$ and consequently $\lim _{\tau \rightarrow+\infty} \frac{N^{\beta-1}}{(1+P)^{\gamma}}=1$, so that, finally, $\lim _{\tau \rightarrow+\infty} P(\tau)=+\infty$.

Summarizing, so far we have proven the following. Suppose a trajectory $\alpha(\tau)$, remaining in $\mathcal{B}^{\prime}$ for all $\tau \geq 0$, starts at a point $Q_{0}=\left(K_{0}, N_{0}, P_{0}\right)$ such that $\dot{K}\left(Q_{0}\right)>0, \dot{N}\left(Q_{0}\right)<0$ and $K_{0} \in(\bar{N}-\varepsilon, \bar{N})$ with $\varepsilon>0$ sufficiently small. Then $\lim _{\tau \rightarrow+\infty} \alpha(\tau)=Q_{\infty}=(\bar{N}, 0,+\infty)$. On the other hand, Lemma 8 states that actually there exist trajectories $\alpha(\tau)$, lying in $\mathcal{B}^{\prime}$ for all $\tau \geq 0$, such that $\lim _{\tau \rightarrow+\infty} \alpha(\tau)=Q_{\infty}$. We also observe that, by the continuous dependence on initial conditions, if a trajectory $\lambda(\tau)$ leaves $\mathcal{B}^{\prime}$ within a finite time $\bar{\tau}$, so do trajectories starting from points sufficiently close to $\lambda(0)$. Hence, on the basis of the previous arguments, if an oscillating trajectory $\alpha(\tau)$ stays in $\mathcal{B}^{\prime}$ for all $\tau \geq 0$, it follows ${ }^{9}$ that there must exist some separatrix trajectory, say $\hat{\alpha}(\tau)$, starting at some point $\hat{Q}_{0}=\left(\hat{K}_{0}, \hat{N}_{0}, \hat{P}_{0}\right)$ and lying in $\mathcal{B}^{\prime}$ for $\tau \geq 0$,

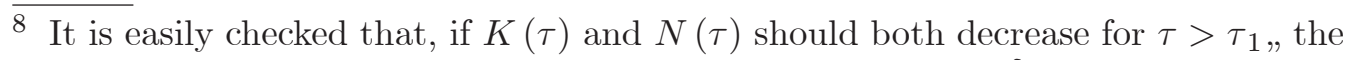
trajectory would reach $K=0$ within a finite time. Just set $K^{\delta}=H$.

9 In fact, suppose, by contradiction, there exists an interval $I=\left(K_{1}, K_{2}\right)$ such that, if $K_{0} \in I$ and $Q_{0}=\left(K_{0}, N_{0}, P_{0}\right)$ satisfies $\dot{K}\left(Q_{0}\right)>0$ and $\dot{N}\left(Q_{0}\right)<0$, then the trajectory $\Gamma(\tau)$ through $Q_{0}$ leaves $\mathcal{B}^{\prime}$ within a finite time. However, when $K_{0}=K_{1}$, there exists $Q_{1}=\left(K_{1}, N_{1}, P_{1}\right)$ satisfying $\dot{K}\left(Q_{1}\right)>0$ and $\dot{N}\left(Q_{1}\right)<0$, such that the trajectory, say $\Gamma_{1}(\tau)$, through $Q_{1}$ remains in $\mathcal{B}^{\prime}$ for all $\tau \in[0,+\infty)$. Then, for a small $\bar{\tau}>0, \Gamma_{1}$ passes through $\bar{Q}_{1}=\left(\bar{K}_{1}, \bar{N}_{1}, \bar{P}_{1}\right)$, with $\bar{K}_{1} \in\left(K_{1}, K_{2}\right), \dot{K}$ $\left(\bar{Q}_{1}\right)>0, \dot{N}\left(\bar{Q}_{1}\right)<0$, therefore leading to a contradiction. 
with $\dot{K}\left(\hat{Q}_{0}\right)>0, \dot{N}\left(\hat{Q}_{0}\right)<0$, reaching at a time $\tau_{1}>0$ a point $Q_{1}=$ $\left(K_{1}, \bar{N}-K_{1},\left(\bar{N}-K_{1}\right)^{-\frac{1-\beta}{\gamma}}-1\right)$. Then, as $\tau_{1}$ is finite, $Q_{1}$ is not a stationary point, which implies, being $\dot{K}\left(Q_{1}\right)=\dot{N}\left(Q_{1}\right)=0, \dot{P}\left(Q_{1}\right) \neq 0$. Moreover, since $\dot{N}(\tau)<0$ for $0<\tau<\tau_{1}, N\left(\tau_{1}\right)$ must be a minimum, i.e., $\dot{P}\left(Q_{1}\right)>0$. It follows, as observed above, that in a right neighborhood of $\tau_{1} K(\tau)$ keeps increasing, so that eventually $\lim _{\tau \rightarrow+\infty} \hat{\alpha}(\tau)=Q_{\infty}=(\bar{N}, 0,+\infty)$, and so do nearby trajectories satisfying analogous conditions, in particular starting from points $Q_{0}$ close to $\hat{Q}_{0}$ with $K_{0}<\hat{K}_{0}$. Then the argument can be repeated, like in the proof of Theorem 4 . Therefore the only possibility for a separatrix $\hat{\alpha}(\tau)$ is to reach a point $Q_{1}$ as above in an infinite time, that is to tend to a stationary point, which cannot be the $\operatorname{sink} \tilde{Q}$, becoming a source for the inverted time $\tau=-t$, but the saddle $Q^{*}$, possibly lying in the boundary of the region $\mathcal{C}$. In fact, inverting again the time, i.e. posing $t=-\tau$, the supposed separatrix $\hat{\alpha}(t)$ should point, as $t>0$, toward values $K(t)<K^{*}$ and $N(t)>N^{*}$ : hence, recalling the proof of Theorem 4 , outside the region $\mathcal{C}$.

In any case, we have proven that $\mathcal{C}$ cannot contain oscillating trajectories lying in $\mathcal{B}^{\prime}$, and therefore in $\mathcal{C}$, for all $t \in(-\infty,+\infty)$. It follows that the only $\omega$-limit set for trajectories of $\mathcal{C}$ is the $\operatorname{sink} \tilde{Q}$.

\section{References}

[1] Alscher, S. 2011. Environmental degradation and migration on Hispaniola Island. Int. Migr. 49, Supplement s1, e164 -e188.

http://dx.doi.org/10.1111/j.1468-2435.2010.00664.x.

[2] Antoci, A., Galeotti, M., Iannucci, G., Russu, P., 2015. Structural change and inter-sectoral mobility in a two-sector economy. Chaos Soliton Fract. 79, 18-29. http://dx.doi.org/10.1016/j.chaos.2015.05.015.

[3] Antoci, A., Russu, P., Sordi, S., Ticci, E., 2014. Industrialization and environmental externalities in a Solow-type model, J. Econ. Dyn. Control 47, 211-224. http://dx.doi.org/10.1016/j.jedc.2014.08.009.

[4] Bade K., 2003. Migration in European History, Wiley-Blackwell, Hoboken, N.J., USA.

[5] Bartolini, S., Bonatti, L., 2002. Environmental and social degradation as the engine of economic growth. Ecol. Econ. 43, 1-16.

http://dx.doi.org/10.1016/S0921-8009(02)00176-3.

[6] Bartolini, S., Bonatti, L., 2003. Undesirable growth in a model with capital accumulation and environmental assets. Environ. Dev. Econ. 8, 11-30. http://dx.doi.org/10.1017/S1355770X03000020. 
[7] Bird, J., 1987. The transferability and depletability of externalities. J. Environ. Econ. Manag. 14, 54-57. http://dx.doi.org/10.1016/0095-0696(87)90005-2.

[8] Boopathi, S., Rameshkumar, M., 2011. Economic and environmental consequences of the impact of industrial pollution: A case experience of domestic rural water supply. Int. J. Ecol. Econ. Stat. 20, 75-94.

[9] Collier, P., 2009. Haiti: from natural catastrophe to economic security. A report for the Secretary-General of the United Nations. Oxford University, Oxford. Available at http://www.securitycouncilreport.org.

[10] Deng, Y., Yang, G., 2013. Pollution and protest in China: environmental mobilization in context. China Quart. 214, 321-336. http://dx.doi.org/10.1017/S0305741013000659.

[11] Dhamodharam, R., Swaminathan, A., 2010. Over-abstraction of ground water and increasing threat of pollution: is farmers' livelihood at stake? A case study in India. Paper presented at world water week in Stockholm, September 2010.

[12] Economy, E. C., 2004. The river runs black: the environmental challenge to China's future. Cornell University Press, Ithaca \& London.

[13] Escofet, A., Bravo-Peña, L. C., 2007. Overcoming environmental deterioration through defensive expenditures: field evidence from Bahía del Tóbari (Sonora, México) and implications for coastal impact assessment. Journal Environ. Manage. 84, 266-273. http://dx.doi.org/10.1016/j.jenvman.2006.06.005.

[14] Gylfason, T., 2001. Natural resources, education, and economic development. Eur. Econ. Rev. 45, 847-859.

[15] Gylfason, T., Herbertsson, T., Zoega, G., 1999. A mixed blessing: natural resources and economic growth. Macroecon. Dyn. 3, 204-225.

[16] Hirsch, F., 1976. The Social Limits to Growth. Harvard University Press, Cambridge, Mass.

[17] Holdaway, J., 2013. Environment and health research in China: The state of the field. China Quart. 214, 255-282. http://dx.doi.org/10.1017/S0305741013000337.

[18] Hueting, R., 1980. New Scarcity and Economic Growth. More Welfare Through Less Production? North Holland, Amsterdam.

[19] Leipert, C., Simonis, U. E., 1988. Environmental damage - environmental expenditures: Statistical evidence on the Federal Republic of Germany. Int. J. Soc. Econ. 15(7), 37-52. http://dx.doi.org/10.1108/eb014111.

[20] Lewis, W. A., 1955. The theory of economic growth, Allen \& Unwin, London.

[21] López, R. E., 2003. The policy roots of socioeconomic stagnation and environmental implosion: Latin America 1950-2000. World Dev. 31, 259-280. 
[22] López, R. E., 2007. Structural change, poverty and natural resource degradation. In: Atkinson, G., Dietz, S., Neumayer, E. (Eds.), Handbook of Sustainable Development. Edward Elgar, Cheltenham, UK.

[23] Lucas R. E., 2004. Life earnings and rural-urban migration, J. Polit. Econ. 112(S1), s29-s59. http://dx.doi.org/10.1086/379942.

[24] Matsuyama, K., 1992. Agricultural productivity, comparative advantage, and economic growth. J. Econ. Theory 58, 317-334. http://dx.doi.org/10.1016/00220531(92)90057-O.

[25] Ranis, G., Fei, J. C., 1961. A theory of economic development. Am. Econ. Rev. 51, 533-565.

[26] Sachs, J. D., Warner, A. M., 1995. Natural resources abundance and economic growth. National Bureau of Economic Research working paper 5398, Cambridge, MA.

[27] Sachs, J. D., Warner, A. M., 1999. The big push, natural resource booms and growth. J. Dev. Econ. 59, 43-76. http://10.1016/S0304-3878(99)00005-X.

[28] Sachs, J. D., Warner, A. M., 2001. The curse of natural resources. Eur. Econ. Rev. 45, 827-838.

[29] Schaefer, M., 1957. Some considerations of population dynamics and economics in relation to the commercial marine fisheries. J. Fish. Res. Board. Can. 14, 669-681.

[30] Shibata, H., Winrich, J. S., 1983. Control of pollution when the offended defend themselves. Economica 50, 425-37. htto://dx.doi.org/10.2307/2554304.

[31] Shogren, J. F., Crocker, T. D., 1991. Cooperative and noncooperative protection against transferable and filterable externalities. Environ. Resour. Econ. 1, 195213.

[32] Solow, R. M., 1956. A contribution to the theory of economic growth. Q. J. Econ. 70, 65-94.

[33] van der Ploeg, F., 2011. Natural resources: curse or blessing? J. Econ. Lit. 49, 366-420. http://dx.doi.org/10.1257/jel.49.2.366.

[34] Yongguan, C., Seip, H. M.,Vennemo, H., 2001. The environmental costs of water pollution in Chongqing, China Environ. Dev. Econ. 6, 313-333. http://dx.doi.org/10.1017/S1355770X01000183.

[35] World Bank, 2007. Cost of pollution in China. Economic estimates of physical damages. Washington DC, USA. 